\title{
Los niveles elevados de estrógenos y testosterona se asociaron a mayor riesgo de cáncer de mama.
}

Elevated Serum Estradiol and Testosterone Concentrations are associated with a high risk for Breast Cáncer. Cauley JA, Lucas FL, Kuller LH, et al. Ann Intern Med. 1999; 130: 270-277.

\section{Objetivo}

Evaluar la hipótesis que las concentraciones séricas de estradiol y testosterona pueden predecir un aumento en el riesgo de desarrollar cáncer de mama.

\section{Diseño}

Estudio caso-control anidado* en un estudio prospectivo de cohorte diseñado para evaluar fracturas osteoporóticas.

\section{Lugar}

Cuatro centros clínicos en EE.UU.

\section{Participantes}

97 casos de cáncer de mama en mujeres mayores de 64 años sin tratamiento de reemplazo estrogénico, y 244 controles seleccionados al azar de la misma cohorte de pacientes.

\section{Evaluación de factores de riesgo}

Los participantes respondieron a un cuestionario al momento de la inclusión evaluando diversos factores de riesgo, entre ellos los relacionados con cáncer de mama.

\section{Medición de resultados principales}

Los casos de cáncer de mama fueron identificados por revisión de las historias clínicas Se midieron las concentraciones de hormonas sexuales al momento de la inclusión en el estudio.

\section{Resultados principales}

La incidencia de cáncer de mama en el grupo de pacientes con menores niveles séricos de estradiol biodisponible y testosterona libres fueron $0,4 / 1000$, mientras que en el grupo con mayores niveles séricos de dichas hormonas fue de 6,5/1000. Comparando con el grupo de menor nivel sérico, el riesgo relativo para desarrollar cáncer de mama en el grupo con niveles más altos de estradiol biodisponible fue de 3,6 (IC 95\%:1,3-10,0) y en el de niveles altos de testosterona de 3,3 (IC $95 \% 1,1-10,3)$.

\section{Conclusiones}

Los niveles séricos de estradiol biodisponible y testosterona parecen jugar un papel en el desarrollo de cáncer de mama. La medición de niveles séricos de estradiol biodisponible y testosterona podrían ser utilizados para estimar el riesgo de desarrollar cáncer de mama.

Fuente de Financiamiento: En parte por el Instituo Nacional de Salud Pública y el U.S. Army Medical Research.

\section{COMENTARIO}

Este trabajo, dentro de un estudio epidemiológico prospectivo de fracturas osteoporóticas, fue realizado para evaluar algo distinto a lo inicialmente pensado, el riesgo de desarrollar cáncer de mama.

El trabajo presenta ciertas limitaciones: inexplicablemente no fue reportado el status de receptores estrogénicos en las pacientes identificadas como portadoras de cáncer de mama, siendo éste uno de los principales factores de riesgo de uso clínico en el manejo de esta enfermedad. Si el estudio pretendía evaluar el riesgo de desarrollar cáncer de mama, una correlación entre niveles séricos de hormonas sexuales y status de receptores estrogénicos hubiese podido aportar una información muy valiosa desde el punto de vista biológico. Tampoco se efectuaron mamografías al momento de inclusión en el estudio de cohorte, que hubiese sido un requerimiento indispensable para cualquier estudio propectivo de cáncer de mama bien diseñado. También resulta curioso que no se haya hecho el esfuerzo de recabar la información de mamografias que seguramente estas mujeres, siendo pacientes añosas, tendrían como control anual.

Este estudio no intenta explorar que es lo que sucede en las mujeres premenopáusicas y contestar esta pregunta sería extremadamente importante. Sabemos que el cáncer tarda probablemente décadas en desarrollarse, por lo tanto sería muy interesante evaluar prospectivamente que sucede en este grupo para, eventualmente, efectuar alguna intervención a fin de reducir el riesgo de desarrollar cáncer de mama.

Básicamente este estudio reporta que los niveles elevados de estradiol biodisponible (porcentaje de estradiol no unido a la globulina transportadora de hormonas sexuales SHBG), y de testosterona libre, se asocian a un riesgo aumentado de desarrollar cáncer de mama.

Estrona, estrona sulfato, testosterona total, dehidroepiandrosterona y androstenediona no se asociaron con un aumento del riesgo de cáncer de mama. En 1998 Hankinson et al. ${ }^{2}$ reportaron en el Journal of The National Cáncer Institute hallazgos que ligaban fuertemente a los niveles de estradiol, estrona, dehidroepiandrosterona, y estrona sulfato con un aumento del riesgo de cáncer de mama. Llamativamente, el estradiol biodisponible y la testosterona no se asociaron con un aumento del riesgo de cáncer de mama, es decir, exactamente lo opuesto que en el estudio que estamos analizando. Al margen de las diferencias técnicas en las mediciones de los niveles hormonales, es im- portante resaltar que muchas veces resulta difícil sacar conclusiones de estu dios retrospectivos, y muchas veces generan más preguntas que respuestas. Este no es el primer estudio que reporta un riesgo aumentado de desarrollar cáncer de mama asociado a niveles séricos aumentados de estradiol, sin embargo curiosamente si es uno de los primeros en reportar que el aumento de niveles de testosterona libre también se asocia con un riesgo aumentado de cáncer de mama. Otros estudios habían reportado aumentos de riesgo concomitantes con aumento de niveles de testosterona pero esta variable no era independiente del aumento del riesgo asociado con el producido por niveles elevados de estradiol. Esto era razonable bajo la hipotesis de que la testosterona podría aumentar el riesgo indirectamente siendo una fuente de estradiol por conversión periférica. Sin embargo este estudio demuestra que el aumento del riesgo asociado a la testosterona es independiente del producido por estradiol. Todo esto es más confuso si recordamos que los andrógenos fueron usados en algún momento como terapia en el cáncer de mama, y actualmente son recomendados como cuarta línea hormonal (cuando todo lo demás falla). Creo que habría que intentar reproducir los datos en un estudio prospectivo bien diseñado antes de concluir que la testosterona es un factor de riesgo importante e independiente del estradiol para desarrollar cáncer de mama. Podríamos asumir que los niveles de hormonas sexuales femeninas se asocian con aumento del riesgo de desarrollar cáncer de mama, sin embargo los datos de la literatura no son suficientemente claros para sugerir que hormona u hormonas deben ser medidas; los datos para la testosterona son aun más confusos, no creo que haya evidencia firme que indique que la testosterona aumentada es un factor de riesgo independiente para desarrollar cáncer de mama. Pero los problemas no terminan aquí aún asumiendo que los niveles de estradiol se asocien con un aumento del riesgo de desarrollar cáncer de mama, no queda claro que se puede hacer con esa información. Los autores sugieren que la medición de los niveles de hormonas sexuales puede ser beneficioso para detectar la población con riesgo aumentado de desarrollar cáncer de mama para eventualmente iniciar tratamiento antiestrógeno. El diseño de este estudio no intenta contestar a la pregunta de si se deberían o no medir los niveles de estrógenos en la población general para detectar un subgrupo de individuos con riesgo aumentado, para contestar a esa pregunta habría que haberla formulado a priori y haber diseñado un estudio prospectivo para contestarla.

Dr. Roberto J. Bitton

Oncólogo Clínico. Coordinador, Programa de Oncología Clínica, ELEA. 